\title{
Instanton size distribution: repulsion or the infrared fixed point?
}

\author{
E.V. Shuryak \\ Department of Physics, \\ State University of New York at Stony Brook, Stony Brook, \\ New York 11794, USA
}

We discuss available information about the instanton size distribution $d(\rho)$, which comes from lattice simulations and the interacting instanton liquid model. Not only they are remarkably consistent, but one can also reproduce $d(\rho)$ with an alternative idea, based on the infrared fixed point. We show, that lattice non-perturbative beta-function (for bare charge renormalization) also suggests such a hint. We also discuss whether it is possible to reconcile the instanton physics with reasonable large-number-of-colors limit, concluding that our fits for $\mathrm{SU}(2)$ and $\mathrm{SU}(3)$ theories are actually very close to the critical value $8 \pi^{2} / g^{2}=N_{c} * 5.4$ when it is the case.

Instantons were discovered 20 years ago [1], but only now their significant role in strong interaction physics is being widely recognized. A large set $(\sim 40)$ of point-to-point correlation functions was calculated in the simplest "instanton liquid" model [2], both numerically [3] and (with certain approximations) analytically [4]. The results are truly spectacular: not only masses and coupling constants of lowest hadronic states (including $\pi, \sigma, \rho, A_{1}, N, \Delta$ and others) are reproduced without additional parameters, but the whole correlation functions happen to be in agreement with those extracted from phenomenology [6] and from lattice simulations [5]. This implies that instanton-induced 't Hooft interaction 11] between light quarks is dominating the interquark forces. Recently, glueballs were added to the list [10], with the conclusion that their specific features in the instanton model (such as small mass and especially small size of the scalar glueball) can quantitatively explain available lattice data.

This agreement is not accidental, as can be seen from the fact that the "instanton liquid" itself was "distilled" from lattice configurations by the "cooling" method [7.9,8]. In spite of the (nearly complete) loss of confinement and of the perturbative gluons, this operation does not affect much these correlators (and hadrons) [9]. Furthermore, the main parameters (the average instantons size $\bar{\rho}$ and instanton spacing $\mathrm{R}$ ) were found to be inside $10 \%$ the same as those suggested in 1982 [2], namely $\bar{\rho} \approx 1 / 3 \mathrm{fm}, R \approx 1 \mathrm{fm}$.

The topic of this work is the shape of the instanton (plus antiinstanton)size distribution $d(\rho)$, related with a number of important issues, both practical and theoretical ones. With all those advances at the phenomenological front, we are still lacking answers to many major questions. One of them, to be discussed in this letter, is: Why are large - size instantons absent in the QCD vacuum? Alternative explanations are: (i) they are suppressed by repulsive interaction between instantons; (ii) the higher-order effects lead to charge renormalization so that their actions are large; (iii) confinement effects screen their gluoelectric fields. In this Letter we will go through this list, and will show that the first two are still strongly competitive.

The first idea is the basis of the 'interacting instanton liquid model' (IILM), formulated as a particular statistical model amenable for numerical simulations [14, 15], its partition function (for $N / 2$ instantons and $N / 2$ antiinstantons) is generally given by

$$
Z=\int \prod_{I=1}^{N} d \Omega_{I} d_{0}\left(\rho_{I}\right) \exp \left[-\sum_{I<J} S_{I J}^{i n t}\right]
$$

where $\Omega_{I}$, denote the orientation, position and the size of pseudoparticle $I, d_{0}(\rho)$ here corresponds to non interacting instantons, with the gluonic interactions included separately. (The quark-related ones are not discussed in this letter.)

The simplest "hard core" model was introduced in [12]. Then it was shown that for the simplest "sum ansatz" 113] such a repulsion actually exists, even slightly stronger than it is needed. Some defects of this ansatz was cured in [14], where the improved trial function known as "ratio ansatz" was proposed: this reproduces phenomenological parameters of the "instanton liquid" well. However, numerical solution [16] of the "streamline" equation 117. have shown that the instanton-antiinstanton valley leads continuously to zero fields. Thus, it seems that the original hopes to stabilize the ensemble at the purely classical level 13] are not fulfilled. Presumably quantum effects (especially subtraction of perturbative contributions, relevant for close instanton-anti-instanton pairs with a strongly attractive interaction) will generate the effective repulsion (see also [18]). Meanwhile, in IILMbased recent studies [10,19] the streamline interaction is supplemented by a repulsive core, with the radius fitted to the value of the gluon and quark condensates.

In Fig.1 (open points) we show the resulting instanton size distribution in IILM, for the pure gauge $\mathrm{SU}(2)$ (a) and $\mathrm{SU}(3)$ (b) theories. In the former case they are compared to first available lattice data [8] (closed points). They are very similar, except at small sizes (instantons with the radius $\rho \sim 1$ in lattice units "fall through the lattice" during "cooling"). At large sizes both lattice data and "interacting liquid" give virtually identical results. 

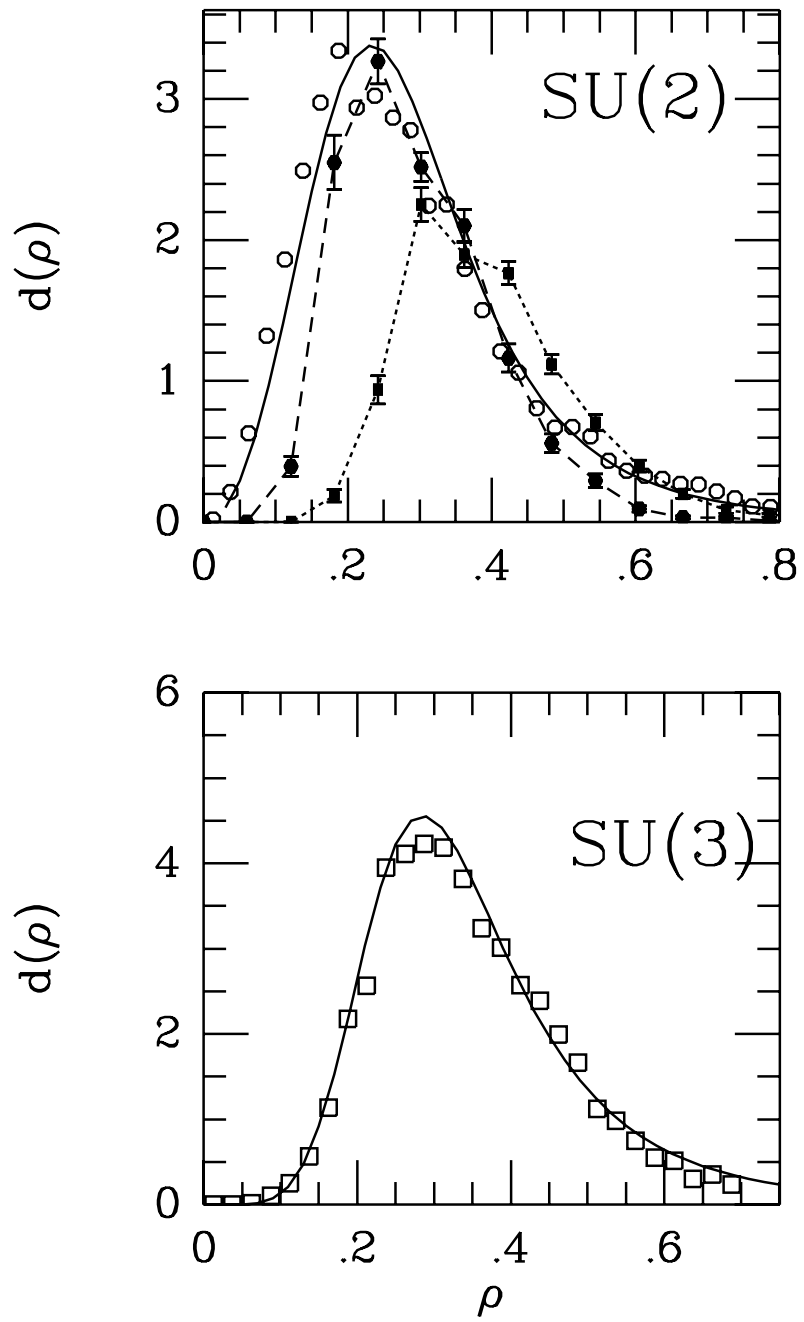

FIG. 1. The instanton size distribution in $\mathrm{d}=4 \mathrm{SU}(2)$ (a) and $\mathrm{SU}(3)$ (b) gauge theories. The open points correspond to interacting instanton liquid", and the closed ones are lattice results By Michael and Spenser, $16^{4}, 4 / g^{2}=2.4$ for squares and $24^{4}, 4 / g^{2}=2.5$ for dots (the dotted and dashed lines just guide the eye). Solid curves correspond to the parametrization discussed in the text. Units are in "femtometers", defined for lattice data by the scalar glueball mass defined as $m_{0^{+}}=1.7$ " $G e V$ "; and by $1 / \Lambda_{P V}$ for the instanton model.

It is well known that (provided the action is large enough $S_{\text {eff }}>>1$ ) one can use the semiclassical theory and derive the following generic expression

$$
d(\rho)=\frac{2 C_{I}}{\rho^{(d+1)}} S^{\left[N_{z m} / 2\right]}(\rho) \exp [-S(\rho)]
$$

where 2 accounts for instantons and anti-instantons, $\mathrm{d}$ is the space-time dimension, and $N_{z m}$ is the number of zero modes ( $4 N_{c}$ for Yang-Mills fields). The value of the constant (which depends on renormalization method) was determined for the Yang-Mills instantons in the classical work by 't Hooft [11]. In order to make our discussion below simpler, we use $C_{I}=0.466 /\left[\left(N_{c}-1\right) !\left(N_{c}-2\right) !\right]$, absorbing the $N_{c}$ dependent factor into a new Lambda parameter $\Lambda_{\text {inst }}=0.632 \Lambda_{\text {Pauli-Villars }}=0.657 \Lambda_{\bar{M} \bar{S}}$.)
Multiple arguments in favor of existence of the infrared fixed point (or "freezing" of the coupling constant) have been many times made before, based on a variety of phenomenological models (one may find a list of references e.g. in a recent paper [20], which came to the same conclusion via analysis of higher-loop corrections to $\sigma\left(e^{+} e^{-} \rightarrow\right.$ hadrons $\left.)\right)$. If so, at large $\rho$ the action is $\rho$-independent, and the following simple prediction follows: $d(r h o) \sim \rho^{-d+1}$, where $\mathrm{d}$ is just the space-time dimension. Are available data in agreement with it?

In terms of statistical accuracy and the widest range studied, the best lattice measurements are those for $\mathrm{d}=2$ $\mathrm{O}(3)$ sigma model [21]. For large sizes the result is $d(\rho) \sim$ $\rho^{-3}$ [8], in perfect agreement with the idea of "frozen" $S(\rho)$.

Furthermore, for the $\mathrm{d}=4 \mathrm{SU}(2)$ gauge theory (see Fig.1(a)) we have fitted the action using the following simple parametrization, a standard two-loop expression for the charge (where $b_{0}=\frac{11}{3} N_{c}, b_{1}=\frac{17}{3} N_{c}^{2}$ )

$$
\frac{8 \pi^{2}}{g^{2}(\rho)}=b_{0} L+b_{1} \log L
$$

with a "regularized" $\log$

$$
L=\frac{1}{p} \log \left[\left(\frac{1}{\rho \Lambda}\right)^{p}+C^{p}\right]
$$

It has two new parameters, $\mathrm{C}$ and $\mathrm{p}$, describing where and how rapidly the "freezing" occurs. Solid line in Fig.1a shows such a fit, with $\Lambda_{\text {inst }}=.66 \mathrm{fm}^{-1}, \mathrm{p}=3.5, \mathrm{C}=4.8$. For the SU(3) gauge theory the data for $d(\rho)$ itself are still missing, but the total instanton density and average size were measured in [9]: $\int d \rho d(\rho) \approx 1.3 \mathrm{fm}^{-4}, \bar{\rho}=0.35 \mathrm{fm}$. One may fix the parameters to reproduce those two integrals: the corresponding solid curve is shown in Fig.1(b). (Parameters in this case are $\Lambda_{\text {inst }}=.70 \mathrm{fm}^{-1}, \mathrm{p}=3.5$, $\mathrm{C}=5.0$. ). Although it is not a fit to open points (the interacting liquid) and is based on different physics, the curve agrees with the points perfectly. Now we plot both fits it in terms of the action (Fig.2a) one can see that it indeed means rather rapid "freezing" of the coupling constants. We do not know why such freezing may occur: but it is remarkable that it should happen where the action itself is large, $S \sim 10-20$ (so that one should not question the semiclassical theory). 

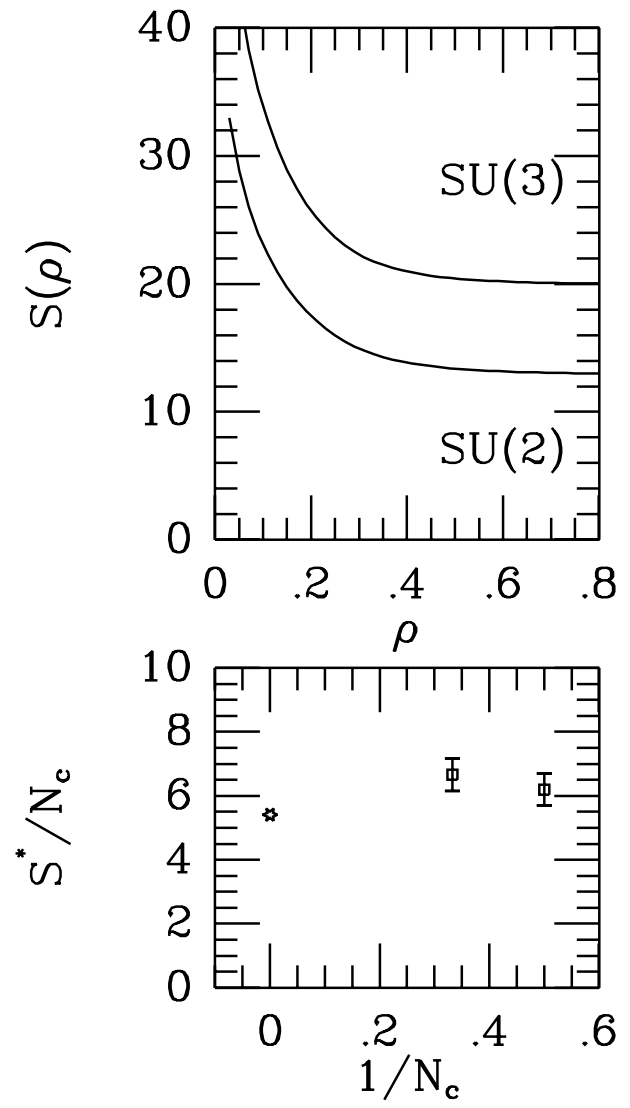

FIG. 2. The upper part shows the instanton action $S(\rho)$ versus $\rho$ (in "fm") according to the parametrization used in Fig.1. The lower part compare the fitted values of the "fixed point" actions $S^{*}$ (divided by the number of colors $N_{c}=2,3$ ) with the critical point in the large $N_{c}$ limit (star).

Let us now comment on the alternative (iii) mentioned above, namely that the cutoff of the large size instantons is related with confinement. Two arguments against its dominance can be given. Although no specific formulae are known (they should depend on the unknown confinement mechanism), the confinement-related correction to the action should have the form $\delta S_{\text {conf }}\left(K \rho^{2}\right)$ where $\mathrm{K}$ is the string tension. Most probably it is a regular function, expandable in powers of its argument: this leads to an exponential cutoff at large $\rho$ rather than power-like one, as suggested by Fig.1. The second: studies of the instanton sizes at non-zero temperatures 22 have recently been made. Near the deconfinement transition (where $K(T) \rightarrow 0$ ) the average instanton size does not grow, while one should expect it to happen (as $1 / K^{1 / 2}$ ) if confinement is involved. (One may investigate this question further by a number of methods: in particular, by checking whether the so called "abelian projected monopole loops" 26] are or are not correlated with instantons in the vacuum ensemble.)

Now, is the existence of the infrared fixed point consistent with non-perturbative beta functions used in the lattice studies? Recall that the bare coupling g(a) is fixed at the input of simulations, then one calculates observables (hadronic masses, etc) and fixes the physical magnitude of the lattice scale a. Reversing the function, one gets the renormalized bare charge g(a), subject for (i) universality test (its independence on the particular observable used) and (ii) comparison with the expected perturbative behaviour ("asymptotic scaling"). The non-trivial fact found (see e.g. 24,25]) is that (i) extends beyond (ii).

A sample of lattice data for the $\mathrm{SU}(3)$ lattice gauge theory, without quarks 24] (open points) and with two massless quark flavors (closed points) [25] shown in Fig.3. Those are usually presented in form of the derivative of $\mathrm{g}(\mathrm{a})$, the famous Gell-Mann-Low beta function. In order to show various theories, one can normalize the derivative to its asymptotic $(g \rightarrow 0)$ perturbative value: thus we plot the following ratio

$$
R_{\beta}(g)=\left[\frac{d\left(1 / g^{2}\right)}{d \log (a)}\right] /\left[\left.\frac{d\left(1 / g^{2}\right)}{d \log (a)}\right|_{g \rightarrow 0}\right.
$$

which tends to 1 the right hand side of Fig.3. As one penetrates into the non-perturbative region the data display a deep drop of $R_{\beta}$ at $6 / g \approx 6$, with subsequent turn upward. Both are very rapid: the latter turn is known in literature as a transition to a "strong coupling" regime. This drop may be an indication for the infrared fixed point, while the upturn is definitely a lattice artifact. (Rapid turnovers are common when a renormalization trajectory is going toward the fixed point, and then misses it closely.) We conjecture that for lattice actions which are better than Wilson one, the corresponding beta function would drop further.

However, even with standard Wilson actions one may investigate other definitions of the renormalized charge, namely those related with the renormalized background fields. This method is quite popular in the perturbative context 28] but (to our knowledge) has not yet been used on the lattice. It is clear which field is the best to try. First, to avoid complications with external current, one should better take a "self-supporting" classical field, such as $D_{\mu} G_{\mu \nu}^{c}=0$. Second, topology adds additional stability: thus instanton is the most natural classical background. Third, its renormalized action depend on one parameter $\rho, S_{\text {eff }}=8 \pi^{2} / g^{2}(\rho)$, and thus this expression can be used to study the non-perturbative charge renormalization. 


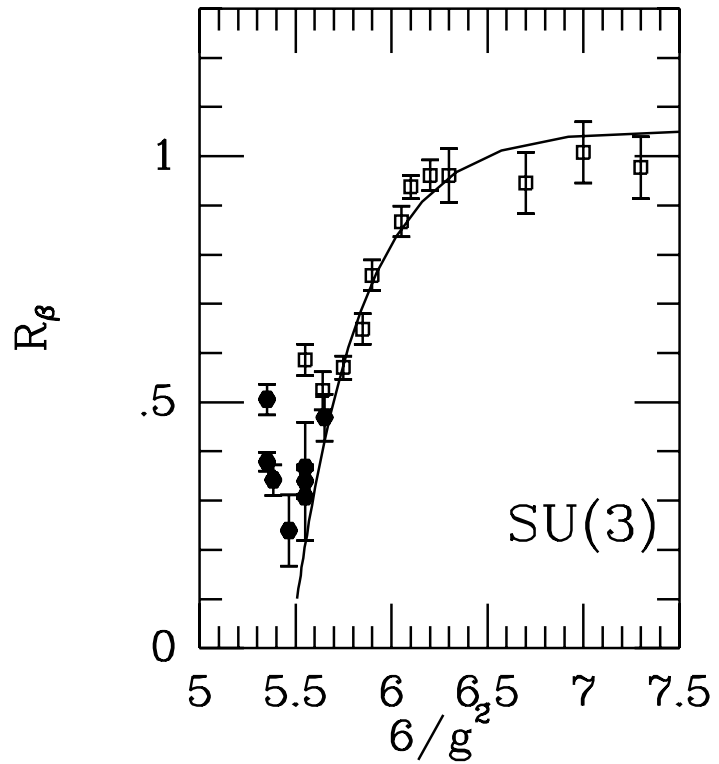

FIG. 3. The non-pertubative beta function for the $\mathrm{SU}(3)$ lattice theory with Wilson action. Open points are taken from R.Gupta review (which is an analysis of the data set from Lapage and Mackenzie), the solid points are from Blum et al. The solid line is a fit discussed in the text.

One can put an instanton on the lattice and then "heat it up", performing standard updates: such studies have been made by DiGiacomo et al [23], establishing renormalization of the topological susceptibility. However, for the proposed goal is not enough to keep a topological charge (as done in ref. 23]): one has to preserve the chosen value of $\rho$. This can be achieved in two ways: (i) while updating a link, one may keep the quantum field $a_{\mu}$ orthogonal to the dilatational zero mode $\delta A_{\mu}^{c} / \delta \rho$ [29]; or (ii) one may use a modified lattice action containing the two-plaquette operators with parameters tuned to make any given size the classical minimum of the lattice action. Either way, the main problem is to get high statistics measurements of the effective action, after subtraction of the usual "average plaquette" is made.

The next topic addressed in this Letter is the fate of instantons in the large $N_{c}$ limit. It was first addressed by Witten [27], who has formulated the following dilemma: either (i) instantons are not dynamically relevant, while all observables have regular $1 / N_{c}$ expansion as suggested by perturbative expansion, or (ii) instantons play some role in the real world, but are exponentially suppressed at large $N_{c}$. Back in 1979, Witten has argued in favor of (i), using analogies with some $\mathrm{d}=2$ models, but today there is no doubt about significance of the instanton-induced effects at $N_{c}=3$. However, it is hard to accept (ii) also, because then all the $1 / N_{c}$ development is undermined.

This dilemma still allows for one loophole [2], an alternative (iii), which allows to reconcile instanton physics with smooth large $N_{c}$ limit. Let us look at its consequences and compare them with the empirical trends found for $N_{c}=2,3$. The semiclassical formula (2) in the large $N_{c}$ limit contains factorial terms in the denominator. The action should grow linearly with $N_{c}$ : $S(\rho)=N_{c} s(\rho)$ and therefore these factorials are exactly cancelled by the gauge zero modes. The next level are the exponential terms, which with our normalization look as follows

$$
d(\rho) \sim \exp \left\{N_{c}[2-s(\rho)+2 \log s(\rho)]\right\}
$$

The limit clearly depends on the sign of the bracket. If there exist the fixed point $s(\rho) \rightarrow s^{*}$, as we advocated above, it is important whether it is larger or smaller than the critical value $s_{c}^{*}=5.4$, the root of the bracket.

Let us compare the values of the fixed actions for $N_{c}=2,3$ obtained from the fits above to the critical value (shown by the star) in Fig.2(b). The first observation is that for two fitted cases the limiting action $S^{*}$ indeed grow linearly with $N_{c}$. Second, the constant is somewhat larger than the critical value, although this is so to say inside the error bars (we have assign those to the points, which relate to the uncertainty due to instanton interactions). In general, it seems quite plausible that at $N_{c} \rightarrow \infty$ instantons will take care of themselves and their density and role will remain roughly constant.

But should we expect the limit $N_{c} \rightarrow \infty$ to be smooth? The answer is negative [13]: at larger $N_{c}$ an "instanton liquid" should become solid, because a growing action (which depends on relative positions) is analogous to a decreasing temperature. Thus, one should expect that both color and translational symmetries would be spontaneously broken. In 15 such phase transition was in fact found for $N_{c}=3$, but at non - physical instanton density (about 60 times the physical one). For larger $N_{c}$ the work is in progress. Lattice studies of theories with $N_{c}>3$ would certainly be of great value.

Finally, let us mention the practical aspect of the studies of $d(\rho)$ : its measurements at small $\rho<0.2 \mathrm{fm}$ is potentially the source of by far the most accurate measurements of $\Lambda_{Q C D}$. As it is well known, here $d(\rho) \sim$ $\Lambda_{Q C D}^{b_{0}} \rho^{\left(b_{0}-d-1\right)}$ : thus even relatively poor accuracy (say $50 \%$, compare to dots at Fig.1a) leads to $\Lambda_{Q C D}$ with accuracy of about $5 \%$, much better than measured today.

In summary, we have argued that existing data for the instanton size distribution can be equally well be described both by the interacting instanton liquid model (IILM) and by the existence of the infrared fixed point. If the second is the case, it seems plausible that this fixed point is not far from the critical point, separating scenarios in which instantons are suppressed or grow in the $N_{c} \rightarrow \infty$ limit. Better measurements of $d(\rho)$ on the lattice (especially at $N_{c}=3$ and larger) are badly needed.

Acknowledgements This work is partially supported by the DOE grant DE-FG02-88ER40388. 
[1] A. A. Belavin, A. M. Polyakov, A. A. Schwartz, and Y. S. Tyupkin, Phys. Lett. 59B, 85 (1975).

[2] E. V. Shuryak, Nucl. Phys. B203, 93, 116 (1982).

[3] E. V. Shuryak and J. J. M. Verbaarschot, Nucl. Phys. B410, 55 (1993); T. Schäfer, E. V. Shuryak, and J. J. M. Verbaarschot, Nucl. Phys. B412, 143 (1994); T. Schäfer and E. V.Shuryak, Phys. Rev. D50, 478 (1994).

[4] D. I. Diakonov and V. Y. Petrov, Nucl. Phys. B272, 259 (1986) 457; M.Hutter,Instantons and meson correlation functions in QCD, hep-ph/9501245.

[5] Chu, M.C. Grandy, J.M. Huang, S. Negele, J.W. Phys.Rev.Lett.70, 225, 1993.

[6] E. V. Shuryak, Rev. Mod. Phys. 65, 1 (1993).

[7] M.I.Polikarpov and A.I.Veselov, Nucl.Phys. B297 (1988) 34.

[8] C.Michael, P.S.Spencer, Instanton size distributions from calibrated cooling, Talk at Lattice-94, Nucl.Phys.(Proc.Suppl.) in press, hep-lat/9411015. Cooling and the $\mathrm{SU}(2)$ instanton vacuum, Liverpool LTH 346

[9] M.-C. Chu, J. M. Grandy, S. Huang, and J. Negele, Phys. Rev. D49, 6039 (94).

[10] T. Schäfer and E.V. Shuryak, Glueballs and Instantons, SUNY-NTG-94-53, Stony Brook 1994.

[11] G. 't Hooft, Phys. Rev. D14, 3432 (1976).

[12] E.-M. Ilgenfritz,M.Muller-Preussker. Phys.Lett.119B(1982)395.

[13] D. I. Diakonov and V. Y. Petrov, Nucl. Phys. B245, 259 (1984).

[14] E.Shuryak, Nucl.Phys.B302:574,599,1988.

[15] E.Shuryak and J.J.M.Verbaarschot, Nucl.Phys.B341 (1990) 1 .

[16] J.J.M.Verbaarschot, Nucl.Phys.B362:33-53,1991

[17] Ya.Ya.Balitsky, A.V.Yung, Phys. Lett. 168B ( 1986) 113119 .

[18] Quantum repulsion between instantons and antiinstantons is derived in a recent work by Diakonov and Petrov,Phys.Rev.D50:266-282,1994 on the basis of unitarity, relating it to the cross sections of multi-gluon processes.

[19] E.Shuryak and J.J.M.Verbaarschot, Screening of the topological charge in a correlated instanton vacuum, SUNY-NTG-94/25, Phys.Rev.D., in press.

[20] A.C.Mattingly and P.M.Stevenson, Phys.Rev.D49 (1994) 437.

[21] Recall that this model is also asymptotically free with the one-loop beta function coefficient $b_{0}=2$. Thus the semiclassical theory leads to $d(\rho) \sim 1 / \rho$ at small $\rho$, or ultraviolet divergent total density and topological susceptibility.

[22] M.-C. Chu and S. Schramm,Instanton content of finite temperature QCD matter,MAP 178, nucl-th/9412016.

[23] Alles, B. Di Giacomo, A. Panagopoulos, H. Vicari, E. Topological charge density renormalization in the presence of dynamical fermions; hep-lat/9501030.

[24] R.Gupta,Scaling, The Renormalization group and improved lattice actions. LA-UR-92-917 Los Alamos, 1992. P.Lepage and P.Mackenzie, Lattice 90, Nucl.Phys.B (Proc.Suppl.)20 (1991) 173.

[25] T.Blum, L.Karkkainen, D.Toussant and S.Gottlieb, The $\beta$ function and EOS for QCD with two flavors of quarks, AZPH-TH/94-22

[26] Shinji Ejiri, Shun-ichi Kitahara, Yoshimi Matsubara and Tsuneo Suzuki; String tension and monopoles in $T \neq 0$ SU(2) QCD; Contribution to Lattice 94, Kanazawa 9428.

[27] E.Witten, Nucl.Phys. B149 (1979) 285.

[28] For example, the first calculation of complete beta functions for a supersymmetric QED and QCD was done using the instanton background: Shifman, M.A. Vainshtein, A.I. Zakharov, V.I. Phys. Lett. 166B ( 1986) 334336 and Sov.J.Nucl.Phys.42:947,1985.

[29] Such algorithm was developed for $\mathrm{d}=2 \mathrm{O}(3)$ sigma model (A.Alejnikov and E.Shuryak, Novosibirsk 1988, unpublished). Furthermore, if one updates more than one link at a time, it is possible to do so keeping orthogonality to all zero modes. 